\title{
Editorial for the Special Issue
}

\author{
Toshio INUI (Guest Editor)
}

Kyoto University, Japan

The cognitive and computational neurosciences have experienced rapid growth over the last decade. For major brain functions such as vision, motor control, and memory, outlines of the responsible neural systems and their computational designs have been elucidated, and detailed knowledge of each system is accumulating day by day. In cognitive science, the processes of higher mental function have been investigated through numerous sophisticated psychological experiments.

For many years, I have been researching visual perception and cognition. About a decade ago, I became interested in human embodiment. Since then, I have come to believe that the mechanisms of visual cognition can be fully understood through considering embodiment.

\section{Embodiment and Two Visual Systems}

The study published by Melvin A. Goodale in 1991 exerted a major influence on subsequent researches in brain science and cognitive science (Goodale, Milner, Jakobson, $\&$ Garey, 1991). He discovered that the functions for appropriate manipulation of an object and for understanding the name or judging the shape of that object are performed by different areas of the brain. While activities of the pathway from the occipital lobe to the frontal lobe of the brain are important for the utilization of knowledge about how to use an object (that is, procedural or functional knowledge), activities of the pathway from the occipital to the temporal lobe are important for the utilization of knowledge about the classification or name of a shape or object. The first pathway is called the dorsal system, and the second is the ventral system.

The dorsal system is involved in information processing concerning use of an object, and represents a "perception for action" system. For example, let us suppose you were to try to drink some water using a cup placed in front of you. To do so, you must reach for and grasp the cup. To accomplish this, you must first accurately gauge the spatial relationship between your hand and the cup (that is, the position of the cup in viewercentered coordinates). You must also devise an appropriate plan to move your finger, hand, and arm. Despite the real complexity of all these processes, humans can usually accomplish such feats without difficulty. We seem to perform these processes automatically and unconsciously, with little more than a simple glance at the cup. That is to say, we do not perceive an object by vision alone; rather, we perceive it with our bodies, and this function is called embodied cognition. Visual and motor signals are coordinated

Correspondence: Professor Toshio Inui, Graduate School of Informatics, Kyoto University, Sakyo-ku, Kyoto 606-8501, Japan (e-mail: inui@i.kyoto-u.ac.jp). 
very well by close interaction between the occipito-parietal cortex and the motor area, allowing us to achieve embodied cognition.

Humans can be considered a well-organized system for acquiring knowledge by interaction with the environment. In such interactions, humans recognize objects within the environment and the actions of other agents in a particular time series. When seeking to understand the mechanisms of knowledge acquisition, insight into human cognitive function through the dynamic interactions between the observer and the environment is critical.

\section{Perception and Memory of Peripersonal and Personal Space}

There are four kinds of 3-D spatial interactions: peripersonal (visuomotor operations in near, body space), focal extrapersonal (visual search and object recognition), action extrapersonal (orienting in topographically defined space), and ambient extrapersonal (orienting in earth-fixed space). Each seems to be associated with a distinct cortical network (Previc, 1998). In peripersonal space, from many different vantage points, we are able to point to an object at a given position using a finger. In addition, we are able to communicate information to others by pointing at the object with a finger. To reach toward and manipulate an object using parts of our physical body, we have to accurately comprehend the viewing direction (visual information) and physical relationships among body parts such as our head, trunk, arms, and hands (body schema). This information allows us to specify appropriate correlations between ourselves and the object within the external environment. We can also memorize large amounts of information about positional relationships between the objects and ourselves. As a neural basis of these functions, it has been suggested that the bimodal neurons in premotor cortex, which have visual and tactile receptive fields, help to guide movements of the head and arms toward or away from nearby stimuli. Furthermore these cells may underlie the ability to reach toward or avoid objects that are no longer directly visible (Graziano, Hu, \& Gross, 1997). Experimental test for split-brain subjects have revealed that abilities to control the use of the hands in a spatial context and to localize environmental stimuli lie within the right inferior parietal lobe, while the abilities to understand visual symbols such as numbers and musical notation, to utilize patterns of hand movements involved in tool usage, and to understand gestures lie in the left inferior parietal lobe (LeDoux, 1982). Recent neuroimaging studies have provided further details regarding the neural mechanisms for these abilities. We thus propose the following outstanding issues:

Q1: What kind of information regarding the physical body and environment do we use, and how do we store such information?

Q2: How is transformation of this information performed on a situational basis?

\section{Action Recognition, Non-Verbal Communication, and the Human Mind}

We communicate not only by means of language, but also using non-verbal methods such as facial expressions, gaze, gestures, and paralinguistics. Nonverbal communication is more fundamental than verbal one. Obviously, the human mind has played (and continues to play) the central role in the survival and continuation of the species. Survival 
has required the development of the following abilities: prediction of the behaviors of others, assessment of novel situations, prediction of future developments, and selection of appropriate behaviors. In day-to-day reality, of course, it is difficult for these abilities to be performed suitably to a given situation. In this regard, facial expressions, gestures, paralinguistics and gaze offer important cues to assess situations.

The rostral part of area 6 in the monkey brain contains neurons called mirror neurons (Rizzolatti, Fadiga, Gallese, \& Fogassi, 1996). Different sets of mirror neurons are triggered by manual behaviors such as the grasping, manipulation, and placement of objects respectively. Activation is also seen when the monkey observes such actions by other (thus the term "mirror neurons"). Furthermore, another set of mirror neurons were also activated selectively by olofacial gestures. This area corresponds to Broca's area in the human brain. The mirror system plays an important role in understanding actions: this system matches an observed action with our own (executed) actions, which is exactly embodiment. This system might also be related to response facilitation (that is, the response facilitated by objects displaying particular attributes and actions by conspecifics), as suggested by Rizzolatti, Fadiga, Fogassi, and Gallese (2002). Observation of both object- and non-object-related actions determined a somatotopically organized activation of premotor cortex in human. Thus, when individuals observe an action, an internal replica of that action is automatically generated in their premotor cortex, the view of which is an extension of mirror system in Broca's area (Buccino et al., 2001).

On the other hand, biological motions and action patterns are visually analyzed in the superior temporal sulcus (STS). In 1997, Leslie Brothers argued that human brain involved an important network especially for social behaviors, which consisted of the STS, amygdala, and orbito-frontal cortex. She referred it as the "social brain". This network visually processes observed actions and estimates emotions in others. In this line of research, several questions present themselves:

Q3: How are action patterns or sequences represented in the brain?

Q4: Does response facilitation occur with observation of actions?

Q5: How do we perform and which part of the neural network is involved in estimation of emotions in another individual based on paralinguistics or actions?

In this special issue of Psychologia, these five particular problems regarding embodiment and nonverbal communication are discussed in six articles.

Finally, I wish to express my deep appreciation to Professor Sakiko Yoshikawa and Ms. Tomoko Kuroda for their patience in editing this issue.

\section{REFERENCES}

Brothers, L. 1997. Friday's footprint: How society shapes the human mind. New York: Oxford University Press.

Buccino, G., Binkofski, F., Fink, G. R., Fadiga, L., Fogassi, L., Gallese, V., et al. 2001. Short communication: Action observation activates premotor and parietal areas in a somatotopic manner: An fMRI study. 
European Journal of Neuroscience, 13, 400-404.

Goodale, M. A., Milner, A. D., Jakobson, L. S., \& Garey, D. P. 1991. A neurological dissociation between perceiving objects and grasping them. Nature, 349, 154-156.

Graziano, M. S. A., Hu, X. T., \& Gross, C. G. 1997. Coding the locations of objects in the dark. Science, 277, 239-241.

LeDoux, J. E. 1982. Neuroevolutionary mechanisms of cerebral asymmetry in man. Brain, Behavior and Evolution, 20, 196-212.

Previc, F. H. 1998. The neuropsychology of 3-D space. Psychological Bulletin, 124, 123-164.

Rizzolatti, G., Fadiga, L., Gallese, V., \& Fogassi, L. 1996. Premotor cortex and the recognition of motor actions. Cognitive Brain Research, 3, 131-141.

Rizzolatti, G., Fadiga, L., Fogassi, L., \& Gallese, V. 2002. From mirror neurons to imitation: Facts and speculations. In A. N. Meltzoff \& W. Prinz (Eds.), The imitative mind (pp. 247-266). Cambridge: Cambridge University Press. 